\title{
Vulnerability and Resilience to Stress and Immune and Neuroendocrine Function in Portuguese Subjects with Psychic Anomaly (Anxiety and Depression)
}

\author{
Eduardo Goncalves ${ }^{1}$, Saul Neves de Jesus ${ }^{2}$ \\ ${ }^{1}$ Department of Psychiatry and Mental Health of Hospital Center of Algarve, Faro, Portugal \\ ${ }^{2}$ Department of Psychology of Faculty of Social and Human Sciences of University of Algarve, Faro, Portugal \\ Email: eduar.goncalves@gmail.com
}

Received 3 August 2015; accepted 12 October 2015; published 15 October 2015

Copyright (C) 2015 by authors and Scientific Research Publishing Inc.

This work is licensed under the Creative Commons Attribution International License (CC BY).

http://creativecommons.org/licenses/by/4.0/

c) (†) Open Access

\begin{abstract}
The present study aimed to investigate the impact of chronic psychosocial stress and resilience, including at a biological level (immune and neuroendocrine function) in Portuguese citizens with psychic anomaly/mental disorder. The sample aggregated 69 participants. It has been used the following psychometric instruments: 21-item depression, anxiety and stress scales (DASS-21), in the Portuguese validated version; measuring state resilience (MSR), in the Portuguese validated version; the Portuguese scale of 23 questions on vulnerability to stress. Serum levels of cortisol, dehydroepiandrosterone sulfate, antibodies anti-viral capsid antigen of Epstein-Barr virus, triglycerides, high density lipoprotein-cholesterol and body mass index have been measured. It has been concluded that factors of vulnerability to stress and chronic stress, of social nature (lack of social support, adverse living conditions), correlate positively with depression, anxiety and stress, and, through alostatic load, are involved in a greater propensity for immune and neuroendocrine dysfunction in this population.
\end{abstract}

\section{Keywords}

Vulnerability to Stress, Stress, Coping, Resilience, Alostatic Load, Anxiety, Depression, Cortisol, Dehydroepiandrosterone, Epstein-Barr Virus, Triglycerides, High Density Lipoprotein-Cholesterol, Body Mass Index 


\section{Introduction (Theoretical Framework)}

\subsection{Social Support, Stress, Appraisal, Coping, Evoked Emotions, Tripartite Model of Anxiety and Depression}

The structural model of Smith and Lazarus (1993) [1] on stress appraisal stands six components of appraisal. Two components of primary appraisal include the motivational relevance, which consists of an assessment of the importance of the situation for the person, and the motivational congruence, that is, the assessment of the extent to which the situation is consistent or not with their current objectives (that is, if it is desirable or undesirable). Four secondary appraisal components include: self-accountability, i.e., the an evaluation of the degree in which the individual himself is responsible for the situation; hetero-accountability, i.e., an evaluation of the degree in which something or somebody is responsible; potential of problem-focused coping, i.e., the perceived competence to act on the situation to maintain or increase its convenience; potential of emotion-focused coping, i.e., the perceived competence for psychological adjustment and to deal with the situation if it is found that has become undesirable. Crossing the outcomes of the two types of appraisal will result in a mapping of these components in terms of the different emotions experienced (as proposed by Smith (1991) [2] and Smith and Lazarus (1993) [1]), and of the major outcomes of appraisals as described by Lazarus and colleagues, in the context of the theory of stress and coping: irrelevance, benefit, challenge, damage, threat [3]. A condition is evaluated as irrelevant to personal well-being if motivational relevance is low. High motivational relevance in combination with motivational congruence (situation appraised as important and desirable) defines the circumstances as beneficial and can evoke feelings of happiness [1] [2]. Beneficial appraisals of self-accountability evoke feelings of pride. Beneficial appraisals of hetero-accountability evoke feelings of gratitude. High motivational relevance appraisals and low motivational congruence (important and undesirable situation) define the circumstances as stressful. Self-accountability appraisals combined with primary appraisals of stress evoke guilt, shame (self-directed negative emotions). Hetero-accountability appraisals combined with primary appraisals of stress evoke anger. Appraisals of high potential of problem-focused coping combined with primary appraisals of stress define circumstances as an opportunity, indicate that the person has potential abilities to change the circumstances in order to make them more syntonic to their wishes and evoke feelings of challenge, determination, that motivate the individual to get involved in the situation and work to make it more desirable [1] [2]. Appraisals of low potential of problem-focused coping combined with primary appraisals of stress define circumstances as damage, indicate that the person is in a precarious situation, for which little can be done to improve and evoke feelings of sadness and resignation, that motivate the person to seek help and possibly to resign on harmful situation, allowing his/her involvement in another one [1] [4]. Appraisals of high potential of emotion-focused coping,. i.e, assessments of competencies to adapt to circumstances which do not function as desired, allow the person to keep calm, facing conditions evaluated as stressful. Appraisals of low potential of emotion-focused coping, i.e, evaluations of incompetence to adapt to circumstances which do not function as desired, define the situation as threat and evoke feelings of fear, anxiety, motivating the person to be vigilant and to take care in an attempt to avoid undesirable results [1]. Stress results from combined high motivational relevance and motivational incongruence, that is, it occurs when a person evaluates their circumstances as important, but undesirable, i.e., stress can be identified as a subjectively important discrepancy between what one wants (motivational state) and what one have in a given situation (situational state), and then it follows that coping consists of individual efforts to reduce the magnitude of this discrepancy. Acting on the circumstances for change in order to put them more in line with wishes corresponds to problem-focused coping [3]. Acting on desires or beliefs so that conditions become more desirable, without change, which can be achieved by strategies as reprioritization objectives [4], evaluate the circumstances in accordance with a more positive perspective, reinterpret the relevance of circumstances with respect to objectives, corresponds to emotion-focused coping, as described by Lazarus and Folkman (1984) [3], and, more appropriately, to accommodative coping, as described by Walker and colleagues (1997) [5].

Subjects with low levels of positive affectivity are characterized by sadness, apathy, anxiety, stress and not rewarding social involvement. The negative affectivity and positive affectivity are not independent. The positive affectivity provides a rupture of stress and supports ongoing efforts to replenish depleted resources from stress [6] [7]. The tripartite model of anxiety and depression, developed by Clark and Watson (1991) [8], proposes that anxiety and depressive disorders overlap considerably by a general, not specific, factor, negative affectivity, which reflects the level of aversive feelings present in an individual. The two remaining factors of this model are 
positive affectivity, which, when low, is relatively specific for depression, and physiological activation, which is relatively specific to anxiety. Lovibond and Lovibond (1995) [9], authors of anxiety, depression and stress scales (DASS), assume that psychological disorders are not categories, that is, the differences between depression, anxiety and stress, experienced by normal subjects and patients, are essentially level ones: depression is characterized mainly by the loss of self-esteem and motivation, and is associated with the perception of low probability of achieving life goals that are meaningful to the individual as a person; anxiety emphasizes the links between persistent state of anxiety and intense fear responses; stress suggests states of excitement and persistent tension, with low resistance to frustration and disappointment.

\subsection{Vulnerability and Resilience to Stress, Anxiety and Depression, and Alostatic Load}

Resilience is a multidimensional phenomenon, defined by Reich, Zautra and Hall (2010) [10] as the result of successful adaptation to adversity. Although the resilient response can be almost universal, it is unlikely that this capacity is equally distributed in population, such as environmental, social, factors that strengthen or weaken the individual resilience to stress [10]. Within an ecological perspective, Holling, Schindler, Walker and Roughgarden (1995) [11] argue that resilience is defined assistem's ability to absorb disturbance prior to the occurrence of fundamental changes in system's state [11]. Intense stress and pain decrease individual ability to distinguish between the presence of positive emotion and the absence of negative emotion, reducing thereby the sustainability of coping strategies concerning positive emotional involvement. According to Reich et al. (2010) [10], psychic and organic homeostasis is sustained, not by emotional neutrality but, by an intentional emotional engagement process. In line with this view, resilience extends beyond the individual ability for recovering from disease's state, and, within this sense, resilience is considered as the amount of stress that a person can withstand without a fundamental change in his/her ability to pursue objectives that give meaning to life. The higher individual ability to stay in a satisfactory life course is, the greater his/her resilience is. However, as resilient recovery relates with passive aspects of resilience, resilience's sustainability meets its proactive, positive, side, that is, for relevant results in the preservation of important commitments and involvements in living tasks (work, leisure and social relationships). Consciousness and choice characterize the development of human values and sustainable objectives [10]. Exceptions to rapid and full recovery, and that challenge homeostatic fundamentals, examples of which, facing stress chronicity, are dysregulation of the hypothalamic-pituitary-adrenal axis, hypercortisolemia or hypocortisolemia, metabolic syndrome, anxiety and depression, present in some people within the concept of alostatic load [12] [13]. Deprivation of means of full understanding of a highly threatening experience that characterizes younger age groups, determines that they are kept not recovered, for years, from emotional after-effects, so early abuse and trauma may later invade the consciousness, interfering in homeostasis, through the determination of a chronic elevation of central operation of psychophysiological processes [14] [15]. Resiliency resources are not positive qualities found in the other end of a single risk continuum but, rather an inseparable factor of global well-being, which gives unique physical and mental benefits, not taken into account through relative risk assessments [16]. The necessary distinction between factors stems from joining several motivational processes, the need for protection and protection against damage, on one hand, and the need to extend individual reach relating to positive objectives, on the other hand [17], being evident the distinct neuronal structures responsible for the regulation of positive emotional responses and for the negative emotional responses [18]. Also, the underlying cognitions of personal control demonstrate the existence of two factors [19], one for agency, optimism and hope, another for helplessness, pessimism and despair. Within research's framework of public health, there have been studied processes taken as buffer's function providers in relation to stress and vulnerability to stress, such as social support [20] and individual control [21] [22]. Rates of risk factors are listed as following (biological, individual, inter-personal/family, community/organizational). Biological ones include: diastolic blood pressure greater than $90 \mathrm{mmHg}$; systolic blood pressure greater than $140 \mathrm{mmHg}$; total cholesterol greater than $240 \mathrm{mg} / \mathrm{dl}$; glucose at rest greater than $124 \mathrm{mg} / \mathrm{dl}$; body mass index (BMI) greater than 25; genetic factors associated with anxiety; elevated C-reactive protein and/or other increases in inflammatory processes. Individual ones include: history of mental disease; depression/helplessness; traumatic brain injury. Inter-personal/familiar ones are: history of childhood trauma/adult abuse; chronic social stress. Community/organizational ones include: presence of environmental accidents; high rates of violent crime; stressful working environment. The corresponding indices of resiliency resources include heart rate variability (HRV), regular physical exercise, 
genetic factors associated with resilience to stress, immune responsiveness and regulation (biological), positive emotional resources, hope/optimism/agency, cognitive functioning, learning/memory, high executive functioning (individual), safe kinship relationships, close social ties (inter-personal/family), green spaces, involvement in activities in a natural environment (for example, community gardening), voluntarism, satisfactory professional life (community/organizational). Active coping strategies, such as planning and problems solving have been associated with a higher degree of well-being and ability to cope with stress, trauma and disease [6]. Denial and behavioral divestment before stressful situations are associated with higher levels of distress [7]. Those subjects with post-traumatic stress disorder avoid coping with trauma-related memories, which contributes to the maintenance of conditioned fear. According to Fredrickson (2001) [23], positive emotions provide a buffer function in relation to the adverse consequences of stress, by decreasing autonomic activation, determined by negative emotions, and increased processes of thought's flexibility (cognitive flexibility) and problem solving. Ability for cognitive revaluation/reappraisal allows reformulation of adverse experiences, through the adduction of positive meaning and mood [23]. Affiliative behaviors, in animals and humans, mitigate the effects of stress, physical injury and infection [24]. High levels of anti-Epstein Barr virus antibodies (EBV-ab) are indicative of decreased cell-mediated immunity that has been related to psychosocial stress. A low function of cell-mediated immunity increases an individual's risk for B-cell lymphoproliferative disorder. Individuals with negative tests for anti-EBV antibodies have increased risk for severe disease associated with EBV, when infected at advanced ages [25]. Repeated or prolonged stress can determine dysregulation or suppression of immune function. Chronic stress suppresses immune-protective parameters such as production of antibodies [26]. The presence of objectives in life, as well as a set of beliefs about what is ethically correct and incorrect, has been associated with resilience. Beliefs and religious or spiritual practices provide, to many people, a structural frame that facilitates recovery and meeting of meaning, upon the occurrence of traumatic or associated with high load stress experiences [27]. Initial adverse living experiences increase the risk of depression and post-traumatic stress disorder in adulthood, through prolonged production of hormones, neurotransmitters and alterations of the central nervous system, which thereafter will affect the resistance to stress [28]. A key factor identified in trained children to overcome adversity is a close relationship with an adult caregiver [29]. Stress at an early age is associated with chronic elevation of corticotrophin releasing hormone levels during adulthood [28]. Changes in the function of the hypothalamic-pituitary-adrenal axis differ according to the specific nature of psychiatric disorder: major depressive disorder is associated with elevated levels of corticotrophin releasing hormone in cerebrospinal fluids and plasma cortisol; post-traumatic stress disorder is associated with elevated levels of corticotrophin releasing hormone and low levels of cortisol [30]. Dehydroepiandrosterone (DHEA), simultaneously secreted with cortisol, in response to stress, may increase stress resistance, protecting against neuronal injury induced by corticosteroids, particularly in the hippocampus resulting from the prolonged activity of the hypothalamic-pituitaryadrenal axis [31]. Higher serum levels of DHEA in patients with post-traumatic stress disorder are associated with lower disease severity and higher recovery of symptomatology. Higher values of DHEA sulphate/cortisol ratios during stress can be protective in healthy individuals [32]. Within the context of the dysfunction of the hypothalamic-pituitary-adrenal axis, several mechanisms have been proposed concerning the evolution towards low cortisol states. A model suggests that, under the influence of chronic stress, initial adaptive response of hypercortisolism, over time, becomes a self-protecting state of hypocortisolism, in order to preserve the endocrine-metabolic system and the brain. Other potential mechanisms of states of hypocortisolism centrally induced include the down-regulation of pituitary receptors of corticotrophin releasing factor, in response to elevations of corticotrophin releasing hormone and to negative feedback, induced by hypercortisolism at central nervous system, over sustained release of stimulating hormones. Relative states of hypocortisolism, or resistance to cortisol, may also occur despite the presence of normal, or even elevated, levels of cortisol [33] [34]. Obesity, an actual public health problem worldwide, in conjunction with metabolic syndrome and the clinical picture of type II diabetes mellitus, integrates the concept of alostatic load [13], which groups several anomalies, including insulin resistance, dyslipidemia and markers of cardiovascular disease. The increased triglycerides (TGL)/high density lipoprotein-cholesterol (HDL) ratio identifies individuals with overweight, insulin resistance, atherogenic particles of low density lipoprotein-cholesterol (LDL), constituting a predictive marker for the presence of atherosclerotic coronary lesions and cardiovascular accident. In addition, low serum HDL levels, in combination with increased serum concentrations of TGL, has predictive value for the development of type II diabetes mellitus [35] [37]. Alostatic load determines long-term effects on cardiovascular system (determining atherosclerosis and car- 
diovascular disease), brain (with decreased neurogenesis and increased dendritic remodeling in the hippocampus, causing loss of the ability to adapt to environmental requirements), adipose tissue and muscle (determining the development of obesity and metabolic syndrome) and immune system (increasing the risk of infection and autoimmune disease) [38]. The mediterranean diet is beneficial in this context, because it is poor in saturated fat (lipid) and rich in monounsaturated fats (consumption of fish and olive oil) and fiber (vegetable consumption). Recent meta-analysis on the effects of the mediterranean diet on metabolic syndrome has highlighted its association with lower values of blood pressure, glucose and triglycerides [39]. Locus coeruleus-norepinephrine system interferes with the functioning of the sympathetic nervous system and the responses of the hypothalamic-pituitary-adrenal axis to stress. Locus coeruleus sustains a general alarm function, in response to potential threats, triggering the release of norepinephrine in amygdala, nucleus accumbens, prefrontal cortex and hippocampus, and its activation also inhibits neurovegetative function, including sleep and eating behavior. A chronically hyperactived locus coeruleus-norepinephrine system predisposes the organism to high levels of anxiety, by inhibition of prefrontal cortex, thus interfering with the regulation of more complex cognitive and emotional processes, and determining cardiovascular clinical problems [40] [41]. Beta-adrenergic receptors blocking in amygdala prevents the development of aversive memories, in animals and humans [42]. Thus, resilience is associated to the reduction of the response of locus coeruleus-norepinephrine system. Serotonin/5-hdroxi-tryptamine (5-HT) has neuro-modulatory effects on other neurotransmitter systems involved in the regulation of mood and anxiety. Stress experiences at an early age can lead to decreased stress tolerance in adult life, through increasing levels of releasing corticotrophin hormone and cortisol, which, in turn, decreases the activity of serotoninergic 5-HT-1A receptors [43]. Dopaminergic neurons are activated by rewarding stimuli and inhibited by aversive stimuli, wherein stress activates dopamine releasing in neurons in medial prefrontal cortex and inhibits its release in nucleus accumbens, a key component of rewarding circuitry. Excessive dopamine release at meso-cortical level after stressful events may be associated with increased vulnerability to stress. Signaling of dopamine facilitates fear extinction, but its role in resilience is not yet totally clarified [44]. Neuronal circuit of fear regulation involves mainly amygdala, hippocampus and ventro-medial prefrontal cortex. Amygdala mediates fear conditioning [45]. Hippocampus mediates contextual and temporal aspects of conditioned fear [46]. Over time, it may occur reactivation and reconsolidation through strengthening, or weakening, by extinction of memories. Amygdala and ventro-medial prefrontal cortex are involved in successful extinction, a process which involves the formation of new memories [47]. The pathophysiology of post-traumatic stress disorder may involve an abnormal fear learning and underlying dysfunction of neural circuitry of fear [48]. Post-traumatic stress disorder patients tend to generalize, from specific conditioned stimuli, to other stimuli in their environment that also become associated with its original trauma, and are therefore fear inductors. Resilience may involve the ability to avoid this generalization of fear. Recently, it was found that, in healthy individuals, the lateral prefrontal cortex, activated by cognitive regulation of emotions can act, through connections of ventro-medial prefrontal cortex with amygdala, in order to decrease fear responses [47]. Phillips, Drevets, Rauch and Lane (2003) [49] described a neuronal model of emotional regulation which comprises a ventral system (integrating amygdala, insula, ventral striatum, ventral anterior cingulate cortex and ventral prefrontal cortex), responsible for the identification of emotional stimuli and production of suitable emotional response, and a dorsal system (including hippocampus, dorsal anterior cingulate cortex and dorsal prefrontal cortex), responsible for the regulation of emotional responses [49]. It has been shown that disturbances of mood and anxiety correlated more consistently with defects in the amygdala, hippocampus, subgenual anterior cingulate cortex and prefrontal cortex [50]. Cognitive reevaluation (reappraisal) is a mechanism by which resilient individuals can reduce or control emotional responses to stressful situations. Recent studies of functional brain imaging, with participants instructed to reappraise the significance of negative images, demonstrate the involvement of medial and lateral prefrontal cortex in the regulation of emotional responses, through top-down control of amygdala activation during cognitive reappraisal [51]. Greater use of cognitive reappraisal in everyday life has also been linked to increased activation of the prefrontal cortex and less activation of the amygdala, in face of negative stimuli [52], suggesting the existence of a possible neuronal mechanism by which cognitive reappraisal increases the resistance to stress. In short, resilience can be related to a greater ability for emotional regulation, while psychopathology may be associated with abnormalities in emotional regulation systems [53]. The rewarding system includes dopaminergic neurons in midbrain, especially in ventral tegmental area, which project in nucleus accumbens and other limbic regions. Recently, it has been highlighted a dysfunction of neural circuitry of rewarding, in patients with major depres- 
sion [54] and post-traumatic stress disorder [55], as well as in young adults with history of abuse in childhood [56]. A large body of epidemiological research suggests that individuals with lack of social support are at greater risk for problems of mental and physical health, and low social support is related to higher levels of depression, anxiety and dissatisfaction with life. The existence of social support is also associated with low mortality from all causes, but particularly due to cardiovascular disease. The interface between social support and processes related with stress is crucial in understanding these epidemiological associations [57]. Research suggests that oxytocin, neuropeptide involved in pro-social behavior and neuroendocrine responses to stress in animals, may mediate some of the protective effects and reducing anxiety that characterize affiliative behaviors [58]. In humans, the combination of social support with oxytocin is more effective in reducing anxiety and reactivity of the hypothalamic-pituitary-adrenal axis in response to psychosocial stress. Oxytocin can also facilitate individual ability of inference of the mental states of others [59]. The ability to empathize may be related to social competence, a characteristic of resilient individuals. The mirror neuron system, working in conjunction with limbic areas, may play a role in understanding the emotions and intentions of others [60].

\section{Empirical Study}

\subsection{Participants}

In the present study, a convenience sampling has been selected, with subjects residents in Olhão, Portugal, referred by general practitioners, to a first psychiatric appointment at the outpatient consultation of psychiatry of hospital of Faro, Portugal, with attributed generic diagnostic hypothesis of anxiety and mood disorders (depression), according to the tenth edition of the international classification of diseases of world health organization [61], with chronic evolution, not subjected to previous interventions of psychopharmacology and psychotherapy. The research protocol has been accepted by the ethics committee of this hospital, and has been in compliance with Helsinki declaration (concerning medical research). Participants provided their written informed consent. The sample is initially composed by 75 elements, $77 \%$ female. The age presents a mean value of 45.3 years (standard deviation: 14.3). Six elements did not answered the measurement scales used, whereby the sample has been reduced to 69 elements.

\subsection{Material and Methods}

The scales have been validated for their use by Portuguese population. It is presented a brief description of the psychometric scales used in this work.

\subsubsection{Questions on Vulnerability to Stress}

The Portuguese scale of 23 questions on vulnerability to stress (23QVS) [62] is an ordinal Likert scale with five possible answers ("0" to " 4 ") between "absolutely agree" and "absolutely disagree" for items 1, 3, 4, 6, 7, 8 and 20 and, conversely, between "absolutely disagree" and "absolutely agree”, for the remaining items. It consists of 23 items, which are organized into seven factors: perfectionism and intolerance to frustration; inhibition and functional dependence; lack of social support; adverse living conditions; dramatization of existence; subjugation; deprivation of affection and rejection. The cutoff point for this scale is greater than 43 (that is, scores higher than 43 mean the existence of vulnerability to stress).

\subsubsection{Items Depression, Anxiety and Stress Scales}

21-items depression, anxiety and stress scales (DASS) [9] [63] is an ordinal Likert scale with four response alternatives (from "0" to " 3 ") between "not applied to me at all" and "applied to me most of the time". It consists of 21 items, which are organized in three dimensions. Seven variables measure appropriately a single dimension, depression, and the cutoff point for depression scale is greater than 7. Seven variables measure appropriately a single dimension, anxiety, and the cutoff point for anxiety scale is greater than 9 . Seven variables measure appropriately a single dimension, stress, and the cutoff point for stress scale is greater than 14. Lovibond and Lovibond (1995) [9] define depression, anxiety and stress as follows: depression includes dysphoria, hopelessness, devaluation, self-depreciation, lack of interest, anhedonia and inertia; anxiety integrates autonomic arousal, skeletal muscle effects, situational anxiety and subjective experience of anxious affect; stress integrates difficulty to relaxing, nervous excitation, easy agitation, irritability/excessive reactivity and impatience. 


\subsection{Measuring State Resilience}

The scale for measuring state resilience(MSR) [64] [65] is an ordinal Likert scale with five possible answers (" 1 " to " 5 ”) between "I totally disagree 'and' I totally agree” and consists of 14 items. The cutoff point is greater than the median, 57.

The departure point of this research focused on the biological impact, particularly at the level of immune and neuroendocrine functions, of vulnerability to stress and chronic stress in patients with psychopathological symptomatology (anxiety and depression). The clinical diagnosis has been confirmed according to the results provided by DASS. In line with technical methodology used at laboratorial medicine unit of hospital of Faro, Portugal, there have been collected morning (08:00 AM) blood samples in peripheral venous blood, for: dosing cortisol (chemical luminescence method); titrating levels of antibodies against viral capsid antigen of EpsteinBarr virus (EBV-ab) (EIA method); determining triglycerides (TGL)/high density lipoprotein-cholesterol (HDL) ratio (enzyme method GPO PAP) (biochemical indicators of vulnerability/risk); titrating levels of dehydroepiandrosteronesulfate (DHEA-S) (chemical luminescence method) (biochemical indicator of protection/resilience factor). It has been calculated body mass index (BMI). For ethical and deontological reasons, it was not considered a subjects-control group (for not requesting diagnostic tests to healthy subjects). This study is descriptive, transversal and quantitative, correlational, not experimental. It has been used Pearson correlation coefficient $r$.

\subsection{Results}

The internal consistency values of all used scales are high, attesting the reliability of the results. Cronbach's alpha coefficient is: 0.864 , for 23QVS; 0.945 , for DASS-depression; 0.886, for DASS-anxiety; 0.899, for DASSstress; 0.838, for MSR. Concerning the existence of disorders in participants, taking as references the cutoff points indicated by the used scales, in $62 \%$ of the subjects there is vulnerability to stress, $54 \%$ exhibit depression, $65 \%$ manifest anxiety, $58 \%$ have stress and $43 \%$ of the participants have resilience. Cortisol levels have an average value of 14.8, with a dispersion of values of 44\% (normal range values: 5 - 25 micrograms per deciliter), $89 \%$ of the elements present normal cortisol levels, $5 \%$ have hypocortisolemia and $6 \%$ have hypercortisolemia (with 5 missing values). DHEA-S levels have an average value of 146.7, with a dispersion of values of $82 \%$ (normal range values: 35 - 430 micrograms per deciliter), 8\% of the elements have values of DHEA-S lower than normal and $3 \%$ have higher than normal values (with 5 missing values). EBV-ab levels have an average value of 67.8 , with a dispersion of values of $54 \%$ (the cutoff point is equal to or greater than 10 Units per milliliter), and $92 \%$ of the elements have values greater than EBV-ab normal values (with 5 missing values). The TGL/HDL ratios have an average value of 2.88 , with a dispersion of values of $104 \%$ (the cutoff point is greater than 3.5), $23 \%$ of the elements have values of TGL/HDL ratios higher than normal (with 4 missing values). The BMI have an average value of 26.1, with a dispersion of values of $20 \%$ (the cutoff point is greater than 25), $46 \%$ of the elements present BMI greater than normal, i.e., overweight (with 2 missing values). In terms of medical comorbidity, 31\% of the participants have endocrine and metabolic disease (predominantly, hypercholesterolemia, diabetes) and hypertension, 40\% have oncological diseases (predominantly, breast cancer, uterine cancer, prostatic cancer), and auto-immune disease (lupus) (29\% of respondents have no comorbidity). When analysis is performed in terms of absolute values, for each variable obtained with the application of 23QVS and biologi$\mathrm{cal} / \mathrm{physiological} \mathrm{measures,} \mathrm{it} \mathrm{is} \mathrm{verified} \mathrm{the} \mathrm{occurrence} \mathrm{of} \mathrm{a} \mathrm{statistically} \mathrm{significant} \mathrm{negative} \mathrm{correlation} \mathrm{between}$ full scale values (vulnerability to stress) and values of EBV-ab, $r=-0.274 ; \mathrm{p}=0.029$. Apart from this, there are levels of statistical significance in the correlations between factor 3 of 23QVS (lack of social support) and cortisol values, $r=-0.248, p=0.048$, between factor 3 of 23QVS (lack of social support) and EBV-ab, $r=-0.264$; $\mathrm{p}=0.035$ and between the factor 4 of 23QVS (adverse living conditions) and EBV-ab, $r=-0.277 ; \mathrm{p}=0.027$, remaining negative. The negative correlation between the DASS dimensions and MSR are related in a statistically significant way $(r=-0.437, \mathrm{p}<0.01$, for the negative correlation between MRS and DASS-depression; $\mathrm{r}=$ $-0.374, \mathrm{p}<0.01$, for the negative correlation between MRS and DASS-anxiety; $r=-0.318$, $p<0.01$, for the negative correlation between MSR and DASS-stress).The 23QVS (vulnerability to stress) and all its dimensions are positively correlated with all dimensions of DASS (depression, anxiety and stress). There have been more strong correlations (above 0.5) between: 23QVS and depression, $\mathrm{r}=0.714, \mathrm{p}<0.001$; 23QVS and anxiety, $\mathrm{r}=$ 0.691, $\mathrm{p}<0.001$; 23QVS and stress, $\mathrm{r}=0.663$, $\mathrm{p}<0.001$; Factor 1 of 23 QVS (perfectionism and intolerance to frustration) and anxiety, $\mathrm{r}=0.558, \mathrm{p}<0.001$; Factor 1 of 23QVS (perfectionism and intolerance to frustration) 
and stress, $\mathrm{r}=0.599, \mathrm{p}<0.001$; Factor 2 of 23QVS (inhibition and functional dependence) and depression, $\mathrm{r}=$ 0.656, $\mathrm{p}<0.001$; Factor 2 of 23 QVS (inhibition and functional dependence) and anxiety, $\mathrm{r}=0.579, \mathrm{p}<0.001$; Factor 2 of 23 QVS (inhibition and functional dependence) and stress, $r=0.554, p<0.001$; Factor 6 of $23 Q V S$ (subjugation) and depression, $\mathrm{r}=0.535, \mathrm{p}<0.001$; Factor 6 of 23QVS (subjugation) and anxiety, $\mathrm{r}=0.547, \mathrm{p}<$ 0.001; Factor 7 of 23QVS (deprivation of affection and rejection) and depression, $r=0.635$, $p<0.001$; Factor 7 of 23QVS (deprivation of affection and rejection) and anxiety. $r=0.647, p<0.001$; Factor 7 of $23 Q V S$ (deprivation of affection and rejection) and stress, $r=0.578, \mathrm{p}<0.001$.

\subsection{Discussion and Conclusions}

In this empirical study, vulnerability to stress correlates positively with depression, anxiety and stress. Also, depression, anxiety and stress correlate negatively with resilience. It has been shown that disturbances of mood and anxiety correlated more consistently with defects in the amygdala, hippocampus, subgenual anterior cingulate cortex and prefrontal cortex [50]. Cognitive reevaluation (reappraisal) is a mechanism by which resilient individuals can reduce or control emotional responses to stressful situations. Recent studies of functional brain imaging, with participants instructed to reappraise the significance of negative images, demonstrate the involvement of medial and lateral prefrontal cortex in the regulation of emotional responses, through top-down control of amygdala activation during cognitive reappraisal [51]. Greater use of cognitive reappraisal in everyday life has also been linked to increased activation of the prefrontal cortex and less activation of the amygdala, in face of negative stimuli [52], suggesting the existence of a possible neuronal mechanism by which cognitive reappraisal increases the resistance to stress. In short, resilience can be related to a greater ability for emotional regulation, while psychopathology may be associated with abnormalities in emotional regulation systems [53].

The lack of social support correlates negatively with serum levels of cortisol. The factor, of vulnerability to stress, lack of social support integrates two dimensions/items (3. When I have problems that bother me I can count on one or more friends to serve me as confidants; 6 . When I have a problem to solve I usually have someone for helping me). A large body of epidemiological research suggests that individuals with lack of social support are at greater risk for mental and physical health problems. The low social support is related to higher levels of depression, anxiety and dissatisfaction with life. The existence of social support is also associated with low mortality from all causes, but particularly due to cardiovascular disease. The interface between social support and processes related stress is crucial in understanding these epidemiological associations [57]. Within the context of the dysfunction of the hypothalamic-pituitary-adrenal axis, several mechanisms have been proposed concerning the evolution towards low cortisol states. A model suggests that, under the influence of chronic stress, initial adaptive response of hypercortisolism, over time, becomes a self-protecting state of hypocortisolism, in order to preserve the endocrine-metabolic system and the brain. Other potential mechanisms of states of hypocortisolism centrally induced include the down-regulation of pituitary receptors of corticotrophin releasing factor, in response to elevations of corticotrophin releasing hormone and to negative feedback, induced by hypercortisolism at central nervous system, over sustained release of stimulating hormones. Relative states of hypocortisolism, or resistance to cortisol, may also occur despite the presence of normal, or even elevated, levels of cortisol [33] [34]. Exceptions to rapid and full recovery, and that challenge homeostatic fundamentals, examples of which, facing stress chronicity, are dysregulation of the hypothalamic-pituitary-adrenal axis, hypercortisolemia or hypocortisolemia, metabolic syndrome, anxiety and depression , present in some people within the concept of alostatic load [12] [13].

The lack of social support and adverse living conditions correlate negatively with serum levels of anti-Epstein-virus antibodies. The factor, of vulnerability to stress, adverse living conditions, integrates two dimensions/ items (4. Often I have enough money to meet my personal needs; 21. The amount of money I have isn't enough to use it in my essential expenses). Repeated or prolonged stress can determine dysregulation or suppression of immune function. Chronic stress suppresses immune-protective parameters such as the production of antibodies [26].

In this study, 23\% of participants present triglyceride (TGL)/high density lipoprotein-cholesterol (HDL) ratio levels higher than normal; 46\% of participants present body mass index (BMI) greater than normal, and, in terms of medical comorbidity, 31\% of the participants have endocrine-metabolic disorder (mainly, hypercholesterolemia, diabetes) and hypertension; 40\% have oncological disease (predominantly, breast cancer, uterine cancer, neoplasia prostate), and auto-immune disease (lupus). Alostatic load determines long-term effects on 
cardiovascular system (determining atherosclerosis and cardiovascular disease), brain (with decreased neurogenesis and increased dendritic remodeling in the hippocampus, causing loss of the ability to adapt to environmental requirements), adipose tissue and muscle (determining the development of obesity and metabolic syndrome) and immune system (increasing the risk of infection and autoimmune disease) [38].

Chronic stress, associated with increased alostatic load, in persons with psychic anomaly/mental disorder may depress immune function (with decreased production of antibodies) and neuroendocrine function (with decreased formation and release of cortisol). This trend is observed in this study, placing subjects with psychic anomaly/mental disorder at risk for infectious, oncologic, autoimmune and endocrine diseases.

\section{References}

[1] Smith, C.A. and Lazarus, R.S. (1993) Appraisal Components, Core Relational Themes and the Emotions. Cognition and Emotion, 7, 233-269. http://dx.doi.org/10.1080/02699939308409189

[2] Smith, C.A. (1991) The Self, Appraisal, and Coping. In: Snyder, C.R. and Forsyth, O.R., Eds., Handbook of Social and Clinical Psychology: The Health Perspective, Pergamon Press, New York, 116-137.

[3] Lazarus, R.S. and Folkman, S. (1984) Stress, Appraisal, and Coping. Springer, New York.

[4] Rasmussen, H.N., Wrosch, C., Scheier, M.F. and Carver, C.S. (2006) Self-Regulation Processes and Health: The Importance of Optimism and Goal Adjustment. Journal of Personality, 74, 1721-1747. http://dx.doi.org/10.1111/j.1467-6494.2006.00426.x

[5] Walker, L.S., Smith, C.A., Garber, J. and Van Slyke, D.A. (1997) Development and Validation of the Pain Response Inventory for Children. Psychological Assessment, 9, 392-405. http://dx.doi.org/10.1037/1040-3590.9.4.392

[6] Southwick, S.M., Vythilingarn, M. and Charney, D.S. (2005) The Psychobiology of Depression and Resilience to Stress: Implications for Prevention and Treatment. Annual Review of Clinical Psychology, 1, 255-291. http://dx.doi.org/10.1146/annurev.clinpsy.1.102803.143948

[7] Carver, C.S. (1997) You Want to Measure Coping but Your Protocol’s Too Long: Consider the Brief COPE. International Journal of Behaviral Medicine, 4, 92-100. http://dx.doi.org/10.1207/s15327558ijbm0401_6

[8] Clark, L.A. and Watson, D. (1991) Tripartite Model of Anxiety and Depression: Psychometric Evidence and Taxonomic Implications. Journal of Abnormal Psychology, 100, 316-336. http://dx.doi.org/10.1037/0021-843X.100.3.316

[9] Lovibond, S.H. and Lovibond, P.F. (1995) Manual for the Depression Anxiety Stress Scales. 2nd Edition, Psychology Foundation of Australia, Sidney.

[10] Reich, J.W., Zautra, A. and Hall, J.S. (2010) Handbook of Adult Resilience. The Guilford Press, New York.

[11] Holling, C.S., Schindler, D.W., Walker, B.W. and Roughgarden, J. (1995) Biodiversity in the Functioning of Ecosystems: An Ecological Synthesis. In: Perrings, C., Maler, L.G., Folke, C., Holling, C.S. and Jansson, B.O., Eds., Biodiversity and Loss: Economic and Ecological Issues, Cambridge University Press, Cambridge, 44-83. http://dx.doi.org/10.1017/cbo9781139174329.005

[12] McEwen, B.S. (1998) Stress Adaptation and Disease: Allostasis and Allostatic Load. Annals of the New York Academy of Sciences, 840, 33-44. http://dx.doi.org/10.1111/j.1749-6632.1998.tb09546.x

[13] McEwen, B.S. and Wingfield, J.C. (2003) The Concept of Allostasis in Biology and Biomedicine. Hormones and Behavior, 43, 2-15. http://dx.doi.org/10.1016/S0018-506X(02)00024-7

[14] Luecken, L.J. and Appelhans, B.M. (2006) Early Parental Loss and Cortisol Stress Responses in Young Adulthood: The Moderating Role of Family Environment. Development and Psychopathology, 18, 295-308. http://dx.doi.org/10.1017/S0954579406060160

[15] Luecken, L.J., Appelhans, B.M., Kraft, A. and Brown, A. (2006) Never Far from Home: A Cognitive-Affective Model of the Impact of Early-Life Family Relationships on Physiological Stress Responses in Adulthood. Journal of Social and Personal Relationships, 23, 189-203. http://dx.doi.org/10.1177/0265407506062466

[16] Steptoe, A., Wardle, J. and Marmot, M. (2005) Positive Affect and Health-Related Neuroendocrine, Cardiovascular, and Inflammatory Processes. Proceedings of the National Academy of Sciences of the United States of America, 102, 6508-6512. http://dx.doi.org/10.1073/pnas.0409174102

[17] Bernston, G.C., Caccioppo, J.T. and Gardner, W.L. (1999) The Affect System Has Parallel and Integrative Processing Components: Form Follows Function. Journal of Personality and Social Psychology, 76, 839-855. http://dx.doi.org/10.1037/0022-3514.76.5.839

[18] Canli, T., Zhao, Z., Desmond, J.E., Kang, E., Gross, J. and Gabrieli, J.D. (2001) An fMRI Study of Personality Influences on Brain Reactivity to Emotional Stimuli. Behavioral Neuroscience, 115, 33-42. http://dx.doi.org/10.1037/0735-7044.115.1.33 
[19] Reich, J.W. and Zautra, A.J. (1991) Experimental and Measurement Approaches to Internal Control in Older Adults. Journal of Social Issues, 47, 143-188. http://dx.doi.org/10.1111/j.1540-4560.1991.tb01839.x

[20] Berkman, L.F. and Glass, T.A. (2000) Social Integration, Social Networks, Social Support, and Health. In: Berkman, L.F. and Kawachi, I., Eds., Social Epidemiology, Oxford University Press, New York, 137-173.

[21] Pearlin, L.I. and Schooler, C. (1978) The Structure of Coping. Journal of Health and Social Behavior, 19, 2-21. http://dx.doi.org/10.2307/2136319

[22] Reich, J.W. and Zautra, A.J. (1990) Dispositional Control Beliefs and the Consequences of a Control-Enhancing Intervention. Journal of Gerontology, 45, 46-51. http://dx.doi.org/10.1093/geronj/45.2.P46

[23] Fredrickson, B.L. (2001) The Role of Positive Emotions in Positive Psychology. The Broaden-and-Build Theory of Positive Emotions. American Psychologist, 56, 218-226. http://dx.doi.org/10.1037/0003-066X.56.3.218

[24] DeVries, A.C., Glasper, E.R. and Detillion, C.E. (2003) Social Modulation of Stress Responses. Physiology and Behavior, 79, 399-407. http://dx.doi.org/10.1016/S0031-9384(03)00152-5

[25] Mihai, A., McDade, T., Williams, S. and Lindau, S. (2008) Blood Spot Measurement of Epstein Barr Virus Antibody Titers in Wave I of the National Social Life, Health \& Aging Project (NSHAP). NORC and the University of Chicago. http://biomarkers.bsd.uchicago.edu/pdfs/TR-EBV.pdf

[26] Dhabhar, F.S. (2011) Effects of Stress on Immune Function: Implications for Immunoprotection and Immunopathology. In: Contrada, R.J. and Baum, A., Eds., The Handbook of Stress Science: Biology, Psychology and Health, Springer Publishing Company, New York, 55.

[27] Pargament, K.I., Smith, B.W., Koenig, H.G. and Perez, L. (1998) Patterns of Positive and Negative Religious Coping with Major Life Stressors. Journal for the Scientific Study of Religion, 37, 710-724. http://dx.doi.org/10.2307/1388152

[28] Heim, C. and Nemeroff, C.B. (2001) The Role of Childhood Trauma in the Neurobiology of Mood and Anxiety Disorders: Preclinical and Clinical Studies. Biological Psychiatry, 49, 1023-1039. http://dx.doi.org/10.1016/S0006-3223(01)01157-X

[29] Masten, A.S., Best, K.M. and Garmezy, N. (1990) Resilience and Development: Contributions from the Study of Children Who Overcome Adversity. Development and Psychopathology, 2, 425-444. http://dx.doi.org/10.1017/S0954579400005812

[30] De Kloet, E.R., Joëls, M. and Holsboer, F. (2005) Stress and the Brain: From Adaptation to Disease. Nature Reviews Neuroscience, 6, 463-475. http://dx.doi.org/10.1038/nrn1683

[31] Morfin, R. and Starka, L. (2001) Neurosteroid 7-Hydroxylation Products in the Brain. International Review of Neurobiology, 46, 79-95. http://dx.doi.org/10.1016/S0074-7742(01)46059-4

[32] Yehuda, R., Brand, S.R., Golier, J.A. and Yang, R.K. (2006) Clinical Correlates of DHEA Associated with PostTraumatic Stress Disorder. Acta Psychiatrica Scandinavica, 114, 187-193. http://dx.doi.org/10.1111/j.1600-0447.2006.00801.x

[33] Edwards, L. and Guilliams, T.G. (2010) Chronic Stress and the HPA Axis: Clinical Assessment and Therapeutic Considerations. The Standard, 9, 1-12. http://www.pointinstitute.org/wpcontent/uploads/2012/10/standard_v_9.2_hpa_axis.pdf

[34] Fries, E., Hesse, J., Hellhammer, J. and Hellhammer, D.H. (2005) A New View on Hypocortisolism. Psychoneuroendocrinology, 30, 1010-1016. http://dx.doi.org/10.1016/j.psyneuen.2005.04.006

[35] Reynolds, K. and He, J. (2005) Epidemiology of the Metabolic Syndrome. The American Journal of the Medical Sciences, 330, 273-279. http://dx.doi.org/10.1097/00000441-200512000-00004

[36] Reaven, G.M. (2005) Insulin Resistance, the Insulin Resistance Syndrome, and Cardiovascular Disease. Panminerva Medica, 47, 201-210.

[37] Laws, A., King, A.C., Haskell, W.L. and Reaven, G.M. (1991) Relation of Fasting Plasma Insulin Concentration to High Density Lipoprotein Cholesterol and Triglyceride Concentrations in Men. Arteriosclerosis, Thrombosis, and Vascular Biology, 11, 1636-1642. http://dx.doi.org/10.1161/01.ATV.11.6.1636

[38] Korte, S.M., Koolhaas, J.M., Wingfield, J.C. and McEwen, B.S. (2005) The Darwinian Concept of Stress: Benefits of Allostasis and Costs of Allostatic Load and the Trade-Offs in Health and Disease. Neuroscience \& Biobehavioral Reviews, 29, 3-38. http://dx.doi.org/10.1016/j.neubiorev.2004.08.009

[39] Kastorini, C.M., Milionis, H.J., Esposito, K., Giugliano, D., Goudevenos, J.A. and Panagiotakos, D.B. (2011) The Effect of Mediterranean Diet on Metabolic Syndrome and Its Components: A Meta-Analysis of 50 Studies and 534, 906 Individuals. Journal of the American College of Cardiology, 57, 1299-1313. http://dx.doi.org/10.1016/j.jacc.2010.09.073

[40] Charney, D.S. (2003) Neuroanatomical Circuits Modulating Fear and Anxiety Behaviors. Acta Psychiatrica Scandinavica, 108, 38-50. http://dx.doi.org/10.1034/j.1600-0447.108.s417.3.x 
[41] Charney, D.S. (2004) Psychobiological Mechanisms of Resilience and Vulnerability: Implications for Successful Adaptation to Extreme Stress. American Journal of Psychiatry, 2, 369-391. http://dx.doi.org/10.1176/foc.2.3.368

[42] McGaugh, J.L. (2004) The Amygdala Modulates the Consolidation of Memories of Emotionally Arousing Experiences. Annual Review of Neuroscience, 27, 1-28. http://dx.doi.org/10.1146/annurev.neuro.27.070203.144157

[43] Lanzenberger, R.R., Mitterhauser, M., Spindelegger, C., Wadsak, W., Klein, N., Mien, L.-K., et al. (2007) Reduced Serotonin-1A Receptor Binding in Social Anxiety Disorder. Biological Psychiatry, 61, 1081-1089. http://dx.doi.org/10.1016/j.biopsych.2006.05.022

[44] Cabib, S., Ventura, R. and Puglisi-Allegra, S. (2002) Opposite Imbalances between Mesocortical and Mesoaccumbens Dopamine Responses to Stress by the Same Genotype Depending on Living Conditions. Behavioural Brain Research, 129, 179-185. http://dx.doi.org/10.1016/S0166-4328(01)00339-4

[45] Delgado, M.R., Olsson, A. and Phelps, E.A. (2006) Extending Animal Models of Fear Conditioning to Humans. Biological Psychology, 73, 39-48. http://dx.doi.org/10.1016/j.biopsycho.2006.01.006

[46] Bast, T. (2007) Toward an Integrative Perspective on Hippocampal Function: From the Rapid Encoding of Experience to Adaptive Behavior. Reviews in the Neurosciences, 18, 253-281. http://dx.doi.org/10.1515/REVNEURO.2007.18.3-4.253

[47] Delgado, M.R., Nearing, K.L., LeDoux, J.E. and Phelps, E.A. (2008) Neural Circuitry Underlying the Regulation of Conditioned Fear and Its Relation to Extinction. Neuron, 59, 829-838. http://dx.doi.org/10.1016/j.neuron.2008.06.029

[48] Yehuda, R. and LeDoux, J. (2007) Response Variation Following Trauma: A Translational Neuroscience Approach to Understanding PTSD. Neuron, 56, 19-32. http://dx.doi.org/10.1016/j.neuron.2007.09.006

[49] Phillips, M.L., Drevets, W.C., Rauch, S.L. and Lane, R. (2003) Neurobiology of Emotion Perception I: The Neural Basis of Normal Emotion Perception. Biological Psychiatry, 54, 504-514. http://dx.doi.org/10.1016/S0006-3223(03)00168-9

[50] Ressler, K.J. and Mayberg, H.S. (2007) Targeting Abnormal Neural Circuits in Mood and Anxiety Disorders: From the Laboratory to the Clinic. Nature Neuroscience, 10, 1116-1124. http://dx.doi.org/10.1038/nn1944

[51] Goldin, P.R., McRae, K., Ramel, W. and Gross, J.J. (2008) The Neural Bases of Emotion Regulation: Reappraisal and Suppression of Negative Emotion. Biological Psychiatry, 63, 577-586. http://dx.doi.org/10.1016/j.biopsych.2007.05.031

[52] Drabant, E.M., McRae, K., Manuck, S.B., Hariri, A.R. and Gross, J.J. (2009) Individual Differences in Typical Reappraisal Use Predict Amygdala and Prefrontal Responses. Biological Psychiatry, 65, 367-373. http://dx.doi.org/10.1016/j.biopsych.2008.09.007

[53] Johnstone, T., van Reekum, C.M., Urry, H.L., Kalin, N.H. and Davidson, R.J. (2007) Failure to Regulate: Counterproductive Recruitment of Top-Down Prefrontal-Subcortical Circuitry in Major Depression. Journal of Neuroscience, 27, 8877-8884. http://dx.doi.org/10.1523/JNEUROSCI.2063-07.2007

[54] Forbes, E.E., Hariri, A.R., Martin, S.L., Silk, J.S., Moyles, D.L., Fisher, P.M., et al. (2009) Altered Striatal Activation Predicting Real-World Positive Affect in Adolescent Major Depressive Disorder. American Journal of Psychiatry, 166, 64-73. http://dx.doi.org/10.1176/appi.ajp.2008.07081336

[55] Sailer, U., Robinson, S., Fischmeister, F.P.S., König, D., Oppenauer, C., Lueger-Schuster, B., et al. (2008) Altered Reward Processing in the Nucleus Accumbens and Mesial Prefrontal Cortex of Patients with Posttraumatic Stress Disorder. Neuropsychologia, 46, 2836-2844. http://dx.doi.org/10.1016/j.neuropsychologia.2008.05.022

[56] Dillon, D.G., Holmes, A.J., Birk, J.L., Brooks, N., Lyons-Ruth, K. and Pizzagalli, D.A. (2009) Childhood Adversity Is Associated with Left Basal Ganglia Dysfunction during Reward Anticipation in Adulthood. Biological Psychiatry, 66, 206-213. http://dx.doi.org/10.1016/j.biopsych.2009.02.019

[57] Uchino, B.N. and Birmingham, W. (2011) Stress and Support Processes. In: Contrada, R.J. and Braum, A., Eds., The Handbook of Stress Science: Biology, Psychology, and Health, Springer Publishing Company, New York, 111-121.

[58] Kosfeld, M., Heinrichs, M., Zak, P.J., Fischbacher, U. and Fehr, E. (2005) Oxytocin Increases Trust in Humans. Nature, 435, 673-676. http://dx.doi.org/10.1038/nature03701

[59] Domes, G., Heinrichs, M., Michel, A., Berger, C. and Herpertz, S.C. (2007) Oxytocin Improves “Mind-Reading” in Humans. Biological Psychiatry, 61, 731-733. http://dx.doi.org/10.1016/j.biopsych.2006.07.015

[60] Schulre-Rurher, M., Markowirsch, H.I., Fink, G.R. and Piefke, M. (2007) Mirror Neuron and Theory of Mind Mechanisms Involved in Face-to-Face Interactions: A Functional Magnetic Resonance Imaging Approach to Empathy. Journal of Cognitive Neuroscience, 19, 1354-1372. http://dx.doi.org/10.1162/jocn.2007.19.8.1354

[61] Medicode (2015) International Classification of Diseases. 10th Edition (ICD-10), World Health Organization (WHO), Geneva.

[62] Vaz-Serra, A. (2000) Construção de uma escla para avaliar a vulnerabilidade ao stress: A 23QVS. Psiquiatria Clínica, 
21, 278-308.

[63] Pais-Ribeiro, J., Honrado, A. and Leal, I. (2004) Contribuição para o estudo da Adaptação Portuguesa das Escalas de Ansiedade, Depressão e Stress (EADS) de 21 itens de Lovibond e Lovibond. Psicologia, Saúde \& Doenças, 5, 229-239. http://hdl.handle.net/10400.12/1058

[64] Hiew, C.C. (1999) Development of a State Resilience Scale. Annual Meeting of the International Council of Psychologists, Salem, 15-19 August 1999.

[65] Martins, M.H.V. (2005) Contribuições para a análise de crianças e jovens em situação de risco-Resiliência e desenvolvimento. Tese de doutoramento, Faculdade de Ciências Humanas e Sociais, Universidade do Algarve, Faro. 